\title{
Produção científica indexada na base Web of Science na área de Neurociências e Comportamento relacionada com o tema Educação
}

\section{Scientific production indexed in the Web of Science in the field of Neuroscience and Behavior related to the theme of Education}

\section{Producción científica indexada en el Web of Science en el campo de Neurociencia y Comportamiento relacionada con el tema de la Educación}

Cristina Haeffner, mestre em Educação em Ciências pela Universidade Federal do Rio Grande do Sul (UFRGS) e bolsista do programa Pronametro, do Instituto Nacional de Metrologia, Qualidade e Tecnologia (Inmetro), Porto Alegre, RS, Brasil. E-mail: chaeffner@yahoo.com.br.

Jorge Almeida Guimarães, doutor em Ciências Biológicas (Biologia Molecular) pela Universidade Federal de São Paulo (Unifesp), professor do Programa de Pós-Graduação em Química da Vida e Ciências da Saúde (PPGQVS) da Universidade Federal do Rio Grande do Sul (UFRGS) e pesquisador do Centro de Pesquisa Experimental do Hospital de Clínicas de Porto Alegre, Porto Alegre, RS, Brasil. E-mail: jguimar@gmail.com.

\section{Resumo}

O presente trabalho faz uma análise cientométrica da área de Neurociências e Comportamento relacionada com o tema Educação, cobrindo dados quali-quantitativos da produção científica no Brasil e no mundo. $O$ estudo abrange o período de 1945 a 2014 para os dados mundiais e, para o Brasil, de 1990 a 2014, além de dados dos grupos de pesquisa do CNPq, e da pós-graduação na área da Capes. Foi possível constatar significativo crescimento do número de artigos publicados 
no mundo e no Brasil. Não obstante, os dados brasileiros são ainda pouco expressivos no número de publicações, no índice de colaboração internacional e no número de grupos de pesquisa, de pesquisadores e de recursos humanos (pós-graduação). Como dado positivo, foi constatada forte interação entre os grupos de pesquisa das instituições brasileiras, o que indica percepção da importância do tema no Brasil.

Palavras-chave: Produção Científica. Neurociências. Educação. Indicadores de Produção Científica.

\section{Abstract}

This work makes a scientometric analysis of Neurosciences and Behavior related to the theme of education, covering qualitative and quantitative data of scientific production in Brazil and worldwide. The study covers the period from 1945 to 2014 for the global data and, for Brazil, from 1990 to 2014. It includes data of the research groups from CNPq and of graduate study in the area from CAPES. It was possible to see significant growth in the number of articles published in the world and in Brazil. Nevertheless, the Brazilian data are still little significant in terms of the number of publications in the international collaboration index and in the number of research groups, researchers and human resources (graduate). On the positive side, a strong interaction between the research groups of Brazilian institutions was found, indicating awareness of the importance of the subject in Brazil.

Keywords: Scientific Production. Neuroscience. Education. Scientific Production Indicators.

\section{Resumen}

En este trabajo se hace un análisis bibliométrico de área de Neurociencias y el Comportamiento relacionado con el tema de educación, incluyendo los datos cualitativos y cuantitativos de la producción científica en Brasil y en todo el mundo. El estudio abarca el período de 
Haeffner e Guimarães / Produção científica indexada na base Web of Science na área de Neurociências e Comportamento relacionada com

1945 a 2014 para los datos globales y de Brasil desde 1990 hasta 2014, y los datos de los grupos de investigación del CNPq, y graduado en el área de la Capes. Era posible ver un crecimiento significativo en el número de artículos publicados en el mundo y en Brasil. Sin embargo, los datos brasileños son todavía poco significativos en el número de publicaciones en colaboración internacional y en el número de grupos de investigación, de los investigadores y de los recursos humanos (graduados). Como dato positivo, se observó una fuerte interacción entre los grupos de investigación de las instituciones brasileñas, lo que indica la conciencia de la importancia del tema en Brasil.

Palabras clave: Producción Científica. Neurociencias. Educación. Indicadores de Producción Científica.

\section{Introdução}

As Neurociências têm recebido, mais recentemente, grande notoriedade entre as áreas científicas em geral, em particular nas Ciências Biológicas. De fato, os avanços alcançados pelos estudos nas Neurociências caracterizaram uma conexão com o chamado século da Biologia ou século do cérebro, levando a destaque os estudos sobre o funcionamento do cérebro. A resultante é a busca pela inserção desses avanços nos processos educacionais, tirando proveito do século do conhecimento. Tal constatação tem induzido pesquisadores a vislumbrar a proximidade entre estudos de Neurociências e de Educação de forma sistemática, enfatizando, sobretudo, a relação ensino-aprendizagem (AMARAL; JANDREY, 2014). Ao longo dos tempos, o desconhecimento sobre os processos fisiológicos que comandam o funcionamento do sistema nervoso central, contido numa "caixa-preta" de difícil acesso, possibilitou a geração de crendices biológicas, como a importância do tamanho do cérebro para uma hipotética diferença no número de neurônios, que seriam elementos diferenciadores da inteligência entre homens e mulheres. Ainda hoje, mitos sobre o cérebro humano, como a crendice há pouco apontada e a de que o ser humano só é capaz de usar $10 \%$ da sua capacidade cerebral, persistem no sistema de ensino, o que acaba sendo, muitas vezes, utilizado para justificar abordagens 
Haeffner e Guimarães / Produção científica indexada na base Web of Science na área de Neurociências e Comportamento relacionada com o tema Educação

ineficazes para a relação ensino-aprendizagem. Muitos desses mitos são distorções de fatos científicos, sendo necessário o estabelecimento de uma maior aproximação entre as áreas de pesquisa para possibilitar melhor comunicação e adequado uso dos conhecimentos gerados pelas Neurociências, visando a sua aplicação no processo educacional (HOWARD-JONES, 2014).

Segundo Amaral e Jandrey (2015), há expectativa quanto a essa aplicabilidade, pois ela está assentada na perspectiva de que os conhecimentos gerados na área de Neurociências guarda alicerce para práticas educacionais eficientes. "A crença de que a fundamentação científica possa garantir o sucesso das práticas educativas não é um fenômeno novo, e sim uma atualização de uma expectativa já presente em outros momentos históricos" (AMARAL; JANDREY, 2015, p. 8). Certamente tais conhecimentos científicos trazem auxílio relevante para o setor educacional, mas, para isso, é necessário que os pesquisadores de ambas as áreas busquem uma compreensão mútua dos seus afazeres, uma vez que, em cada área, são praticados métodos próprios e formas distintas de gerar conhecimento.

Assim, vê-se como necessária e premente a formação de profissionais da educação com conhecimentos de neurociência, pois, embora sejam evidenciadas muitas relações entre as duas áreas de conhecimento, seus desdobramentos práticos para formação docente ainda são tímidos (DE PONTES VIEIRA, 2013). Nesse sentido, vale a observação de Bartoszeck (2006), que considera que têm sido poucas as publicações da área de Neurociências com foco na atividade educacional e que, por isso mesmo, se faz necessário fazer a ligação entre a Neurociência e a sala de aula, de modo a proporcionar uma direção efetiva e passível de ser empregada na prática do ensino-aprendizagem. É sabido que principalmente a área de Neurociência Cognitiva faz progressos rápidos em conhecimentos altamente relevantes para a educação, mas, ainda assim, existe um abismo entre a ciência atual e suas aplicações em sala de aula. Existe, nas escolas, uma necessidade latente de se obterem informações sobre o cérebro, e os professores estão ansiosos para colher os benefícios do século do cérebro e aplicálos em seus alunos (GOSWAMI, 2006). 
Haeffner e Guimarães / Produção científica indexada na base Web of Science na área de Neurociências e Comportamento relacionada com

Por sua vez, muitos pesquisadores mostram-se convictos de que a área de Neurociências está cada vez mais conectada com a noção de que as práticas educacionais voltadas para os processos de ensinar e de aprender podem orientar e beneficiar pesquisas sobre o cérebro. Métodos aplicados às Neurociências ampliaram a compreensão da mente de forma altamente relevante para a prática educacional. Portanto, quando se questiona sobre o quanto a área de Neurociências pode ser útil para a educação é insuficiente se basear apenas na compreensão unidirecional sobre a função cerebral. Assim, como recomendado por Sigman et al. (2014), os neurocientistas devem estar cientes de que a neurociência é um campo de pesquisa atual de grande relevância e com forte apelo comercial e, portanto, eles têm a responsabilidade de disseminar uma visão rigorosa dos resultados, de forma a apoiar firmemente o diálogo com outras áreas.

James J. Heckman (2007, 2011), tomando por base suas observações sobre habilidades cognitivas e socioemocionais, pesquisas que the garantiram o Prêmio Nobel de Economia no ano 2000, afirma que existe uma grande harmonia entre Economia, Neurociências e Psicologia do Desenvolvimento. Para chegar a tal concepção, ele analisou os resultados obtidos nos últimos anos por neurocientistas, cujos estudos permitiram, por experiências bioquímicas, genéticas e fisiológicas apropriadas, avanços no conhecimento sobre como agem os circuitos neurais que controlam comportamentos cognitivos, emocionais, sociais e educacionais. Foi, então, possível inferir que investimentos em estudos educacionais que forneçam resultados capazes de desenvolver adequadamente as habilidades cognitivas e da personalidade acabam substanciando a melhoria da produtividade socioeconômica dos indivíduos, da sociedade e das nações.

No contexto desta análise e em sintonia com a visão de vários autores, conclui-se que iniciativas que consigam aproximar as áreas de Neurociências e de Educação podem orientar políticas educacionais de longo prazo e fazem sentido para subsidiar procedimentos capazes de melhor reforçar, com base em evidências experimentais, o processo de acompanhamento do desenvolvimento socioemocional das crianças e adolescentes. Esse aspecto se torna mais relevante, na medida em que 
Haeffner e Guimarães / Produção científica indexada na base Web of Science na área de Neurociências e Comportamento relacionada com o tema Educação

psicólogos, neurocientistas, educadores e pais continuam buscando superar os desafios da educação infantil. No centro desse esforço, a busca pela melhoria da educação das crianças deve continuar a ser a motivação maior para a ação construtiva e formar a base para as políticas públicas, visando alcançar avanços na qualidade da educação em todos os níveis.

A conexão Neurociência-Educação passa a constituir um novo paradigma centrado na percepção de como a ciência pode subsidiar a formulação de políticas sociais mais amplas, inclusivas e colaborativas com outras disciplinas. Expostas desde a primeira infância e mesmo ainda na fase uterina aos avanços do século do conhecimento e neles inseridas, as crianças se mostram altamente receptivas e habilitadas a aprender melhor e mais rápido, constituindo uma geração digital, como demonstram dia a dia no contato com a realidade que as cerca e é facilmente perceptível. A escola necessita, assim, acompanhá-las e se capacitar para obter melhor proveito de tais oportunidades. Como propõem Carew e Magsamen (2010), certamente essas circunstâncias produzirão uma sociedade mais bem equipada para o futuro, e a parceria Neurociência-Educação constitui uma oportunidade ideal para produzir soluções educativas baseadas em evidências experimentais capazes de guiar o processo educacional do século XXI (CAREW; MAGSAMEN, 2010).

Valendo-se da importância desse contexto, no presente artigo buscou-se identificar as características quali-quantitativas ${ }^{1}$ da produção científica na área de Neurociências e Comportamento conectada com a Educação, o que configura uma temática de estudo nova, identificável pelas publicações no Brasil e no mundo, e, bem assim, sua comparação com outros países. Tais informações permitem uma visão geral sobre as perspectivas de utilização dos conhecimentos científicos gerados e a inserção dos pesquisadores atuando na área em cada país. Foi,

Neste estudo foram considerados indicadores qualitativos o percentual de documentos citados e o Fator de Impacto (FI), e indicadores quantitativos 0 número de documentos e 0 percentual de colaboração internacional. então, efetuado um levantamento atualizado do desempenho científico mundial, extraindo das bases de dados as informações sobre os indicadores de produção científica indexada internacionalmente. Foram identificados os principais países e instituições onde se desenvolve pesquisa nessa temática e, para os dados do Brasil, além da análise dos indicadores das publicações, foram levantados os dados sobre a força 
Haeffner e Guimarães / Produção científica indexada na base Web of Science na área de Neurociências e Comportamento relacionada com

de trabalho na pesquisa nacional na área, utilizando os indicadores fornecidos pelo Diretório dos Grupos de Pesquisa do Conselho Nacional de Desenvolvimento Científico e Tecnológico (CNPq) e ainda as informações sobre a formação de recursos humanos via pós-graduação na área.

\section{Procedimentos metodológicos}

O trabalho se caracteriza como um estudo cientométrico descritivo, que faz da geração e da utilização das informações científicas uma investigação quali-quantitativa, com o propósito de contribuir com uma melhor concepção dos dados da atividade científica como uma atividade social (MEIS; LETA, 1996), uma vez que a cientometria “[...] permite o tratamento e gerenciamento das informações procedentes de bases de dados científicas, bem como uma análise de categorias e/ ou variáveis dentro do universo estudado a fim de que possa contribuir para o desenvolvimento da ciência atual" (HAEFFNER, 2006, p. 31).

Conforme Silva e Bianchi (2001), pode-se concluir que a cientometria está associada com a demografia da comunidade científica mundial e tem se tornado um tema importante em países mais industrializados, como também naqueles em desenvolvimento que pretendem melhorar a distribuição dos seus fundos de suporte à ciência. Sendo assim, governos e centros de pesquisa têm mostrado interesse em aplicar esse conhecimento para manipular mais apropriadamente os recursos de fomento, utilizando os indicadores científicos na busca por estimar a capacidade científica, tecnológica e de inovação nos respectivos países.

O estudo aqui apresentado tem como proposta comparar os dados de produção científica da área de Neurociências e Comportamento que possuem relação com a temática da Educação. Foram analisados, em âmbito mundial, dados de 1945 a 2014 e, no que se refere ao Brasil, do período de 1990 a 2014. A diferença de período se deve ao fato de o Brasil não ter nenhuma publicação indexada nessa temática antes de 1990, enquanto os dados mundiais incluem publicações desde 1945, 
Haeffner e Guimarães / Produção científica indexada na base Web of Science na área de Neurociências e Comportamento relacionada com o tema Educação

ano de início de indexação da base utilizada. O estudo inclui também informações sobre grupos de pesquisa e dados da pós-graduação no Brasil.

Os indicadores da produção científica foram extraídos da base Web of Science Core Collection, uma das maiores e mais completas base de dados on-line pertencente à Thomson Reuters e disponivel por intermédio do Portal de Periódicos da Capes. A base é composta pelas coleções:

- Science Citation Index Expanded (SCI-EXPANDED), que indexa publicações das diversas áreas de 1945 até o presente:

- Social Sciences Citation Index (SSCI), que indexa publicações das Ciências Sociais de 1956 até o presente;

- Arts \& Humanities Citation Index (A\&HCI), que indexa publicações das áreas de 1975 até o presente;

- Conference Proceedings Citation Index - Science (CPCI-S), que indexa citações das publicações de 1990 até o presente; e

- Conference Proceedings Citation Index Social Science \& Humanities (CPCl-SSH), que indexa citações das publicações de 1990 até o presente.

Para a metodologia de busca, no primeiro momento, no campo de pesquisa avançada, utilizou-se o rótulo WC (Web of Science Category), que inclui as seguintes subcategorias: Neuroscience, Neuroimaging, Clinical Neurology, Behavioral Sciences e Biological Psychology, restringindo o tipo de documento por artigos, notas ou artigos de revisão, no período de 1945 (ano de início de indexação de publicações na base) até o ano de 2014. O agrupamento das informações dessas cinco subcategorias corresponde à área de Neurociências e Comportamento da base ESI (Essencial Science Indicators), conforme apresentado na Tabela 1. 
Tabela 1. Dados coletados

\begin{tabular}{|l|c|c|}
\hline \multirow{2}{*}{ Categorias da Web of Science } & \multicolumn{2}{|c|}{$\mathbf{N}^{\circ}$ de documentos } \\
\cline { 2 - 3 } & Total & Com o termo educat \\
\hline Neuroscience & 853.299 & 5.201 \\
\hline Clinical Neurology & 543.799 & 10.770 \\
\hline Behavioral Sciences & 153.583 & 1.415 \\
\hline Biological Psychology & 65.892 & 225 \\
\hline Neuroimaging & 41.659 & 250 \\
\hline \multicolumn{1}{|c|}{ Total } & $\mathbf{1 . 6 5 8 . 2 3 2}$ & $\mathbf{1 7 . 8 6 1}$ \\
\hline Total sem dupla contagem & $\mathbf{1 . 3 5 2 . 4 9 5}$ & $\mathbf{1 4 . 6 6 0}$ \\
\hline
\end{tabular}

Fonte: Web of Science, Thomson Reuters. Acesso em: 10 maio 2015.

No período 1945-2014 foram publicados 1.658.232 artigos distribuídos nas cinco subcategorias. Esse total embute uma dupla contagem de $18,5 \%$ referente a artigos que foram contados duplicadamente por terem sido publicados em revistas indexadas em mais de uma subcategoria.

Dentro desse conjunto de dados foi feita a busca por "educat*" no campo TS (Tópico), que pesquisa pelo termo nos seguintes campos de um registro: Título, Resumo, Palavras-chave do autor e Keywords Plus, que são palavras-chave atribuídas pela equipe da Thomson Reuters responsável pela base. Excluída a dupla contagem (aproximadamente 18\%) de artigos publicados em revistas de mais de uma subcategoria, foram encontrados 14.660 documentos, que foram, então, analisados quanto aos campos: ano de publicação do documento, nome do periódico, país e instituição do autor. Os documentos foram exportados para o InCites, base também pertencente à Thomson Reuters, que tabula tais dados, sendo possivel analisar os indicadores de interesse. Segue uma breve descrição dos indicadores (THOMSON REUTERS, 2015) utilizados neste trabalho:

a) Número de documentos: somatório do número total de artigos, artigos de revisão e notas, indexados na base Web of Science;

b) Percentual de colaboração internacional: percentual de publicações que possuem coautores de dois ou mais países; 
Haeffner e Guimarães / Produção científica indexada na base Web of Science na área de Neurociências e Comportamento relacionada com o tema Educação

c) Percentual de documentos citados: corresponde ao número de documentos citados, dividido pelo número total de documentos;

d) Fator de impacto: medida que reflete o número de citações de artigos publicados em determinado periódico dividido pelo total de artigos.

Os dados quantitativos referentes aos grupos de pesquisa foram extraídos do Diretório dos Grupos de Pesquisa no Brasil, de responsabilidade do CNPq, que disponibiliza informação dos grupos de pesquisa científica e tecnológica em atividade no país. Na consulta parametrizada da base corrente, no campo Termo de busca foram inseridos os termos "neuro*" e "educ*”, e, na opção Aplicar busca nos campos, foram marcados: nome do grupo, nome da linha de pesquisa e palavra-chave da linha de pesquisa. Foram recuperadas as informações relativas ao número de grupos de pesquisa, de pesquisadores e de estudantes, todas selecionadas, uma a uma, no "Consultar por".

Para os dados da pós-graduação, a busca foi realizada no GeoCapes e na Plataforma Sucupira. Foi feita a extração dos programas de pós-graduação com o termo "neuro" no nome, na área de concentração e/ou na linha de pesquisa em funcionamento. Não foram encontradas informações sobre cursos de pós-graduação vinculando totalmente Neurociências e Educação. Devido a isso, a busca foi feita utilizando-se o termo "educ"* nos sites desses programas, e nenhuma informação foi recuperada; então, a pesquisa foi realizada na descrição (em seus respectivos sites) do programa, das disciplinas e das linhas ou projetos de pesquisa, com o uso das palavras "aprendizado", "aprendizagem", "neuroplasticidade”, "neuroeducação" e "cognição", no entendimento de que têm uma ligação com a educação, termo que não foi encontrado.

Depois de obtidos, todos os dados foram organizados em figuras e tabelas a fim de que fossem estabelecidas as correlações necessárias para a análise. Como instrumento operacional para tabulação dos dados, foi usada planilha eletrônica do Exce/ do Microsoft Office. 
Produção científica em Neurociências e Comportamento com o termo

"educação"

A busca combinada de Neurociências e Comportamento com o termo "educação" resultou em números relativamente baixos nos dados de produção científica mundial quando comparados com os dados da categoria Neurociências e Comportamento como área indexada na Web of Science. A Figura 1 mostra, todavia, que há um crescimento contínuo na evolução da produção científica. Tais dados permitem calcular que a proporção de artigos que vinculam os termos "neurociências" e "educação" em relação aos dados totais da categoria Neurociências e Comportamento, vem crescendo consistentemente a cada quinquênio, na seguinte sequência: 1990-1994, 0,45\%; 1995-1999, 1,1\%; 2000-2004, 1,5\%; 2005-2009, 2,1\% e 2010-2014, 2,5\%. Os dados confirmam que, apesar de constituir tema de pesquisa relativamente novo, a combinação Neurociências e Comportamento com Educação constitui subárea de pesquisa já estabelecida com crescimento contínuo e relevante.

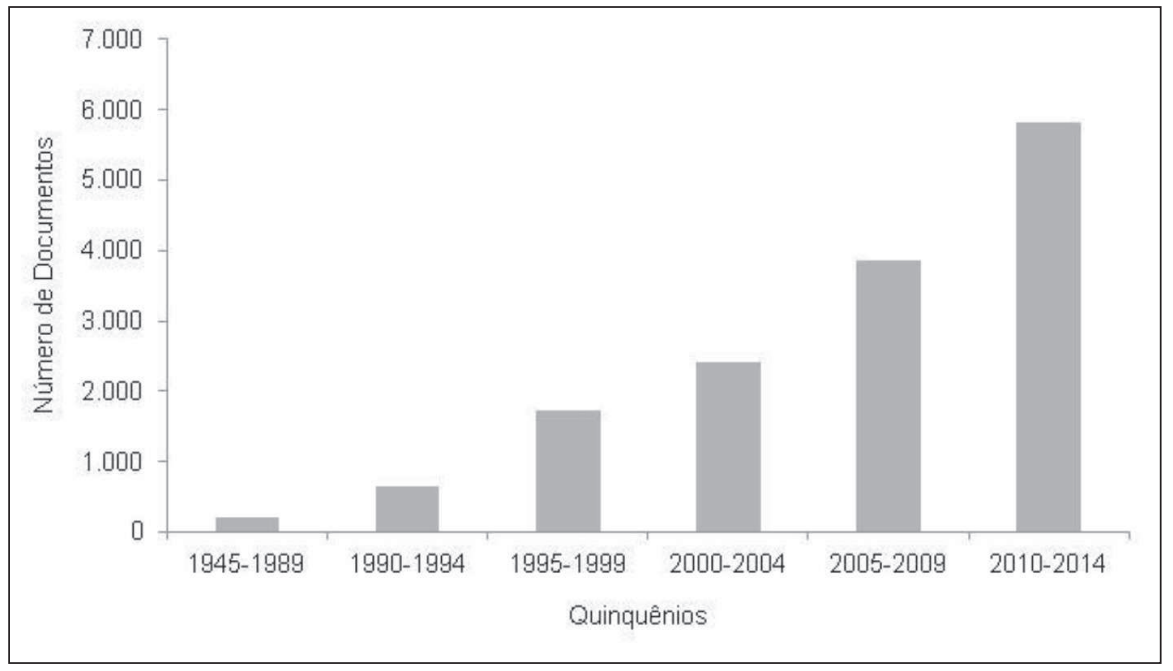

Figura 1. Mundo: produção científica em Neurociências com artigos vinculados ao tema Educação

Fonte: Web of Science, Thomson Reuters. Acesso em: 10 maio 2015

No Brasil, embora com números mais baixos, também se verifica crescimento contínuo da produção de artigos em Neurociências vinculados ao tema Educação (Figura 2). Os cálculos da proporção da 
Haeffner e Guimarães / Produção científica indexada na base Web of Science na área de Neurociências e Comportamento relacionada com o tema Educação

subcategoria sequencialmente: $1 \%$ nos dois primeiros quinquênios, passando, no seguinte, para $1,4 \%$ e chegando a $2,7 \%$ nos dois últimos períodos.

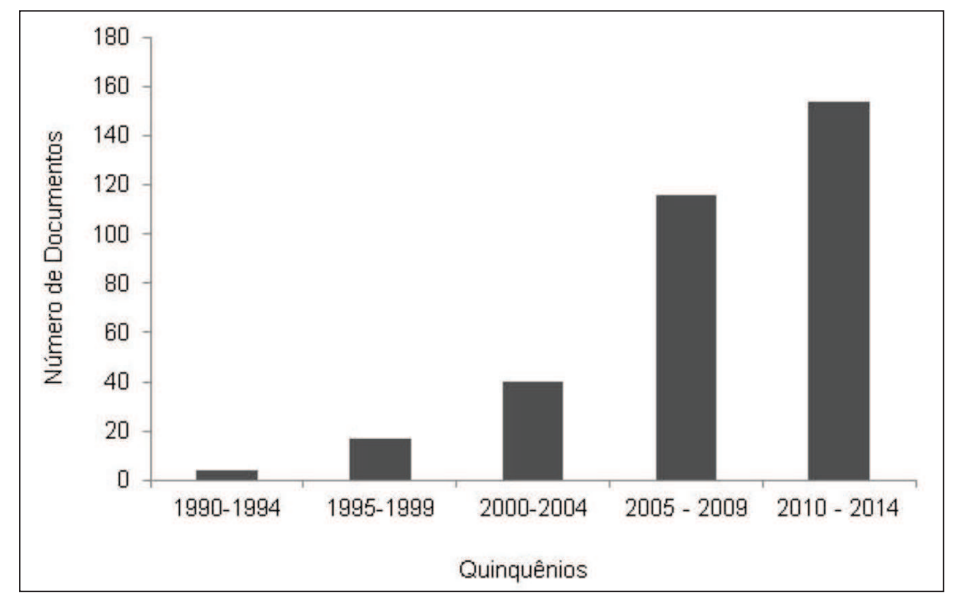

Figura 2. Brasil: produção científica em Neurociências com artigos vinculados ao tema Educação

Fonte: Web of Science, Thomson Reuters. Acesso em: 10 maio 2015

De fato, o índice de crescimento da produção científica na área de Neurociências e Comportamento com a temática Educação no Brasil chega a ser maior do que o crescimento verificado na produção mundial a partir do quinquênio 2000-2004 (Figura 3).

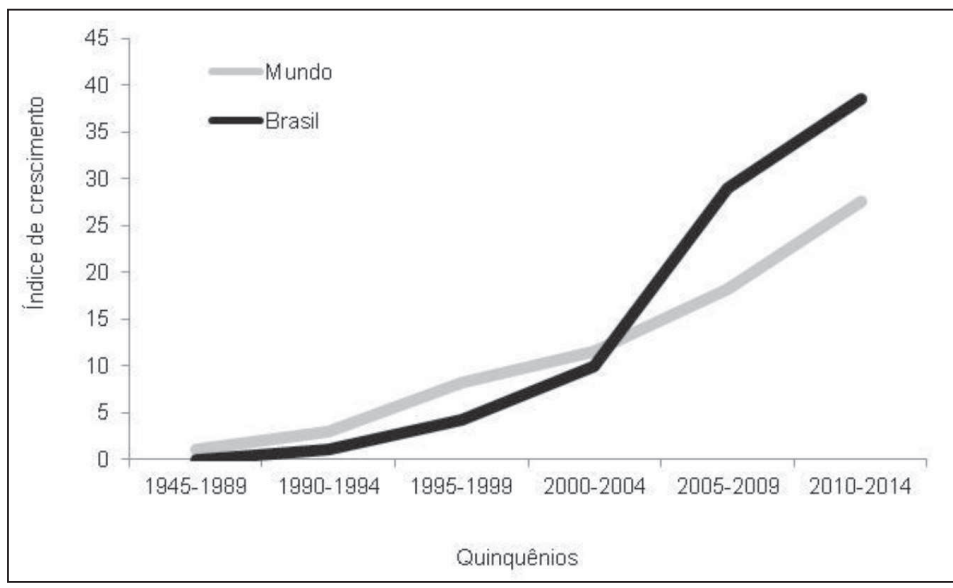

Figura 3. Índice de crescimento da produção científica em Neurociências e Comportamento com artigos vinculados ao tema Educação Comparação Brasil x Mundo

Fonte: Web of Science, Thomson Reuters. Acesso em: 10 maio 2015 
No conjunto, os dados das Figuras 2 e 3 indicam que há positivo interesse por parte da comunidade científica brasileira da área no que se refere à influência que os conhecimentos recentemente obtidos em Neurociências vêm demonstrando exercer sobre a capacidade de aprendizagem das crianças.

No ranking por país (Tabela 2), o Brasil fica na $13^{a}$ posição, à frente de diversos países com muito mais larga experiência em pesquisas em Neurociências e Comportamento e em outras áreas científicas e possuidores de qualificados e reconhecidos mundialmente sistemas educacionais. A média do percentual de colaboração internacional (artigos que possuem um ou mais autores com afiliação em distintos países) é de 39,3\%. No entanto, diversos países (Suíça, Nova Zelândia, Irlanda, Áustria, Bélgica, Dinamarca, Portugal, Suécia e Inglaterra) apresentam índices de $28 \%$ a $74 \%$ mais altos do que a média dos 35 países da tabela. Verifica-se ainda que o Brasil se situa no grupo dos dez países com menores percentagens (menos de 30\%) de colaboração internacional na área, ou seja, mais de 70\% dessa produção é feita por autores afiliados somente a instituições brasileiras.

Tabela 2. Ranking da produção científica na área de Neurociências e Comportamento com artigos vinculados ao tema Educação-1945 a 2014

\begin{tabular}{|c|l|c|c|}
\hline Rank. & \multicolumn{1}{|c|}{ Países } & N $^{\circ}$ de Documentos & \% Colaboração internacional \\
\hline 1 & EUA & 6.401 & 19,4 \\
\hline 2 & Inglaterra & 1.026 & 50,4 \\
\hline 3 & Canadá & 1.016 & 41,2 \\
\hline 4 & Alemanha & 954 & 35,8 \\
\hline 5 & França & 721 & 38,1 \\
\hline 6 & Itália & 718 & 38,8 \\
\hline 7 & Austrália & 669 & 38,0 \\
\hline 8 & Holanda & 637 & 38,3 \\
\hline 9 & Espanha & 515 & 32,6 \\
\hline 10 & China & 375 & 44,7 \\
\hline 11 & Suécia & 372 & 51,9 \\
\hline 12 & Japão & 368 & 17,9 \\
\hline 13 & Brasil & $\mathbf{3 3 1}$ & $\mathbf{2 8 , 5}$ \\
\hline
\end{tabular}


Haeffner e Guimarães / Produção científica indexada na base Web of Science na área de Neurociências e Comportamento relacionada com o tema Educação

\begin{tabular}{|c|c|c|c|}
\hline Rank. & Países & $\mathbf{N}^{\circ}$ de Documentos & \% Colaboração internacional \\
\hline 14 & Turquia & 245 & 10,2 \\
\hline 15 & Finlândia & 241 & 36,3 \\
\hline 16 & Suíça & 233 & 68,4 \\
\hline 17 & Noruega & 219 & 37,6 \\
\hline 18 & Coreia do Sul & 202 & 24,8 \\
\hline 19 & Israel & 192 & 39,3 \\
\hline 20 & Escócia & 184 & 36,4 \\
\hline 21 & Índia & 178 & 26,0 \\
\hline 22 & Bélgica & 169 & 57,4 \\
\hline 23 & Taiwan & 155 & 27,1 \\
\hline 24 & Dinamarca & 152 & 52,3 \\
\hline 25 & Grécia & 136 & 32,6 \\
\hline 26 & Áustria & 134 & 60,5 \\
\hline 27 & Portugal & 98 & 52,0 \\
\hline 28 & Irlanda & 73 & 61,1 \\
\hline 29 & Cingapura & 70 & 45,7 \\
\hline 30 & Polônia & 68 & 37,3 \\
\hline 31 & Argentina & 67 & 47,8 \\
\hline 32 & República Tcheca & 66 & 27,7 \\
\hline 33 & Rússia & 65 & 26,2 \\
\hline 34 & Nova Zelândia & 63 & 61,9 \\
\hline 35 & País de Gales & 63 & 32,3 \\
\hline \multicolumn{2}{|c|}{ Outros Países } & 946 & --- \\
\hline \multicolumn{2}{|c|}{ Total Mundo } & 18.122 & --- \\
\hline
\end{tabular}

Fonte: Web of Science, Thomson Reuters. Acesso em: 10 maio 2015

\section{Periódicos e instituições}

A Tabela 3 lista as 35 principais revistas que publicam artigos em Neurociências e Comportamento vinculados com a temática em Educação. Desse conjunto, 15 são editadas nos Estados Unidos, outras 18 em países europeus (entre elas, seis na Holanda e cinco na Inglaterra) e uma no Japão. Destaque-se, na lista, a revista brasileira Arquivos de Neuro-Psiquiatria, que ocupa a $33^{\text {a }}$ posição, com um total de 113 
artigos $(34,1 \%)$ do total brasileiro no período. 0 fator de impacto dessas revistas tem uma larga variação (de 0,83 a 8,3) sendo a mediana e a média da tabela de 2,92 e 3,21, respectivamente. Neurology, editada nos Estados Unidos, é a revista com maior destaque tanto no total de artigos publicados como também no fator de impacto. Verifica-se ainda elevada e não usual proporção de artigos citados (91\% na média) para todas as revistas da tabela.

Tabela 3. Número de documentos por revista na área de Neurociências e Comportamento com artigos vinculados ao tema Educação, publicados no mundo no período 1945 a 2014

\begin{tabular}{|c|c|c|c|c|c|}
\hline Rank. & Periódicos & País & $\mathbf{N}^{\circ}$ Doc. & $\begin{array}{l}\% \text { Doc. } \\
\text { citados }\end{array}$ & $\begin{array}{l}\text { Fator de } \\
\text { Impacto }\end{array}$ \\
\hline 1 & Neurology & EUA & 678 & 90,0 & 8,286 \\
\hline 2 & Journal of Affective Disorders & Holanda & 316 & 90,0 & 3,383 \\
\hline 3 & Stroke & EUA & 306 & 94,0 & 5,723 \\
\hline 4 & $\begin{array}{l}\text { Journal of Pain and Symptom } \\
\text { Management }\end{array}$ & EUA & 298 & 94,0 & 2,795 \\
\hline 5 & Epilepsy \& Behavior & EUA & 281 & 89,0 & 2,257 \\
\hline 6 & Archives of Neurology & EUA & 245 & 98,0 & 7,419 \\
\hline 7 & $\begin{array}{l}\text { Journal of Clinical and Experimental } \\
\text { Neuropsychology }\end{array}$ & EUA & 245 & 93,0 & 2,083 \\
\hline 8 & Clinical Neuropsychologist & Irlanda & 233 & 86,0 & 3,097 \\
\hline 9 & $\begin{array}{l}\text { Journal of the International } \\
\text { Neuropsychological Society }\end{array}$ & EUA & 217 & 93,0 & 2,963 \\
\hline 10 & Spine & EUA & 210 & 91,0 & 2,297 \\
\hline 11 & Brain Injury & Inglaterra & 205 & 91,0 & 1,808 \\
\hline 12 & Epilepsia & EUA & 205 & 90,0 & 4,571 \\
\hline 13 & $\begin{array}{l}\text { Dementia and Geriatric Cognitive } \\
\text { Disorders }\end{array}$ & Suíça & 195 & 96,0 & 3,547 \\
\hline 14 & $\begin{array}{l}\text { Journal of Developmental and } \\
\text { Behavioral Pediatrics }\end{array}$ & EUA & 192 & 90,0 & 2,129 \\
\hline 15 & Appetite & Holanda & 190 & 86,0 & 2,691 \\
\hline 16 & $\begin{array}{l}\text { Journal of Intellectual Disability } \\
\text { Research }\end{array}$ & Inglaterra & 177 & 89,0 & 1,788 \\
\hline 17 & $\begin{array}{l}\text { Developmental Medicine and Child } \\
\text { Neurology }\end{array}$ & Inglaterra & 167 & 98,0 & 3,510 \\
\hline 18 & $\begin{array}{l}\text { Journal of Neurology Neurosurgery } \\
\text { and Psychiatry }\end{array}$ & Inglaterra & 167 & 98,0 & 6,807 \\
\hline 19 & $\begin{array}{l}\text { Alzheimer Disease \& Associated } \\
\text { Disorders }\end{array}$ & EUA & 162 & 93,0 & 2,440 \\
\hline 20 & Journal of Alzheimers Disease & Holanda & 154 & 87,0 & 4,151 \\
\hline
\end{tabular}


Haeffner e Guimarães / Produção científica indexada na base Web of Science na área de Neurociências e Comportamento relacionada com o tema Educação

\begin{tabular}{|c|c|c|c|c|c|}
\hline Rank. & Periódicos & País & $\mathbf{N}^{\circ}$ Doc. & $\begin{array}{l}\% \text { Doc. } \\
\text { citados }\end{array}$ & $\begin{array}{l}\text { Fator de } \\
\text { Impacto }\end{array}$ \\
\hline 21 & $\begin{array}{l}\text { Journal of Nervous and Mental } \\
\text { Disease }\end{array}$ & EUA & 150 & 87,0 & 1,688 \\
\hline 22 & Revista de Neurologia & Espanha & 148 & 85,0 & 0,830 \\
\hline 23 & $\begin{array}{l}\text { Encephale-Revue de Psychiatrie } \\
\text { Clinique Biologique et Therapeutique }\end{array}$ & França & 145 & 75,0 & 0,698 \\
\hline 24 & Neurosurgery & EUA & 141 & 86,0 & 3,620 \\
\hline 25 & European Journal of Neurology & Inglaterra & 138 & 93,0 & 4,055 \\
\hline 26 & Pain & Holanda & 135 & 99,0 & 5,213 \\
\hline 27 & Neuropsychologia & Holanda & 125 & 96,0 & 3,302 \\
\hline 28 & Neuroepidemiology & Suíça & 123 & 93,0 & 2,580 \\
\hline 29 & Sleep & EUA & 123 & 94,0 & 4,591 \\
\hline 30 & $\begin{array}{l}\text { Psychiatry and Clinical } \\
\text { Neurosciences }\end{array}$ & Japão & 119 & 92,0 & 1,634 \\
\hline 31 & Journal of the Neurological Sciences & Holanda & 116 & 90,0 & 2,474 \\
\hline 32 & Nervenarzt & Alemanha & 114 & 80,0 & 0,787 \\
\hline 33 & Arquivos de Neuro-Psiquiatria & Brasil & 113 & 88,0 & 0,843 \\
\hline 34 & $\begin{array}{l}\text { Journal of Head Trauma } \\
\text { Rehabilitation }\end{array}$ & EUA & 112 & 96,0 & 2,920 \\
\hline 35 & Journal of Neurology & Alemanha & 110 & 95,0 & 3,377 \\
\hline \multicolumn{3}{|c|}{ Outros títulos de periódicos } & 7.910 & - & - \\
\hline \multicolumn{3}{|c|}{ Dados da Tabela } & 6.755 & 91,0 & 3,210 \\
\hline
\end{tabular}

Fonte: Web of Science, Thomson Reuters. Acesso em: 10 de maio 2015.

Na Tabela 4 podemos visualizar o número de artigos de autores brasileiros, publicados nas 30 principais revistas do mundo, sendo de observar que apenas uma revista é publicada no Brasil, a já mencionada Arquivos de Neuro-Psiquiatria. A tabela mostra que, do total de artigos publicados pelos autores brasileiros no período 1990-2014 (331 documentos), essas revistas publicaram a grande maioria (75\%) do total dos artigos brasileiros. Quanto aos indicadores de índice de citações $(79,7 \%)$, fator de impacto $(2,92)$ e mediana dos fatores de impacto das revistas (2,69), não há diferenças acentuadas em relação às revistas da Tabela 3. 
Tabela 4. Número de documentos de autores brasileiros por revista na

área de Neurociências e Comportamento com artigos vinculados ao tema Educação no período 1990 a 2014

\begin{tabular}{|c|c|c|c|c|c|}
\hline Rank. & Periódicos & País & $\mathbf{N}^{\circ}$ Doc. & $\begin{array}{l}\% \text { Doc. } \\
\text { Citados }\end{array}$ & $\begin{array}{l}\text { Fator de } \\
\text { Impacto }\end{array}$ \\
\hline 1 & Arquivos de Neuro-Psiquiatria & Brasil & 106 & 87,0 & 0,843 \\
\hline 2 & Journal of Affective Disorders & Holanda & 20 & 85,0 & 3,383 \\
\hline 3 & Epilepsy \& Behavior & EUA & 12 & 100 & 2,257 \\
\hline 4 & Neurology & EUA & 8 & 100 & 8,286 \\
\hline 5 & $\begin{array}{l}\text { Alzheimer Disease \& Associated } \\
\text { Disorders }\end{array}$ & EUA & 7 & 100 & 2,440 \\
\hline 6 & $\begin{array}{l}\text { European Archives of Psychiatry and } \\
\text { Clinical Neuroscience }\end{array}$ & Alemanha & 7 & 100 & 3,525 \\
\hline 7 & Headache & EUA & 7 & 57,0 & 2,707 \\
\hline 8 & Revista de Neurologia & Brasil & 7 & 68,0 & 0,830 \\
\hline 9 & Acta Neurologica Scandinavica & Dinamarca & 5 & 76,0 & 2,395 \\
\hline 10 & Appetite & Holanda & 5 & 70,0 & 2,691 \\
\hline 11 & Cephalalgia & Noruega & 5 & 52,0 & 4,891 \\
\hline 12 & Cognitive and Behavioral Neurology & EUA & 5 & 80,0 & 0,946 \\
\hline 13 & Sleep Medicine & Holanda & 5 & 100 & 3,154 \\
\hline 14 & Cerebrovascular Diseases & EUA & 4 & 75,0 & 3,754 \\
\hline 15 & $\begin{array}{l}\text { Dementia and Geriatric Cognitive } \\
\text { Disorders }\end{array}$ & Suiça & 4 & 75,0 & 3,547 \\
\hline 16 & Journal of Alzheimers Disease & Holanda & 4 & 100 & 4,151 \\
\hline 17 & Stroke & EUA & 4 & 100 & 5,723 \\
\hline 18 & Aphasiology & Inglaterra & 3 & 33,0 & 1,530 \\
\hline 19 & Brain Injury & Inglaterra & 3 & 67,0 & 1,808 \\
\hline 20 & $\begin{array}{l}\text { Journal of Pain and Symptom } \\
\text { Management }\end{array}$ & EUA & 3 & 100 & 2,795 \\
\hline 21 & $\begin{array}{l}\text { Journal of the International } \\
\text { Neuropsychological Society }\end{array}$ & EUA & 3 & 100 & 2,963 \\
\hline 22 & Journal of the Neurological Sciences & Holanda & 3 & 100 & 2,474 \\
\hline 23 & Movement Disorders & EUA & 3 & 100 & 5,680 \\
\hline 24 & Seizure-European Journal of Epilepsy & Inglaterra & 3 & 67,0 & 1,822 \\
\hline 25 & Spine & EUA & 3 & 100 & 2,297 \\
\hline 26 & Applied Animal Behaviour Science & Holanda & 2 & 50,0 & 1,691 \\
\hline 27 & Brain and Cognition & EUA & 2 & 100 & 2,477 \\
\hline 28 & British Journal of Neurosurgery & Inglaterra & 2 & 50,0 & 0,960 \\
\hline 29 & Childs Nervous System & Alemanha & 2 & 0,0 & 1,114 \\
\hline 30 & Epilepsia & EUA & 2 & 100 & 4,571 \\
\hline \multicolumn{3}{|c|}{ Outros títulos de periódicos } & 82 & - & - \\
\hline \multicolumn{3}{|c|}{ Dados da Tabela } & 249 & 79,7 & 2,92 \\
\hline
\end{tabular}

Fonte: Web of Science, Thomson Reuters. Acesso em: 10 maio 2015. 
Haeffner e Guimarães / Produção científica indexada na base Web of Science na área de Neurociências e Comportamento relacionada com o tema Educação

A Tabela 5 mostra o ranking da produção científica das instituições na área de Neurociências e Comportamento com vínculo com o tema Educação. As 50 instituições listadas na tabela publicaram 10.173 artigos, o equivalente a 57\% do total mundial (17.861 artigos Tabela 1) no período 1945-2014. Assim como ocorre nos periódicos, a grande maioria das instituições (38), majoritariamente universidades, está radicada nos Estados Unidos e produziu 77,5\% (7.888) dos artigos da tabela. A única brasileira entre estas 50 instituições é a Universidade de São Paulo, na $45^{\text {a }}$ posição, com 122 artigos (1,12\% da tabela e 37\% da produção brasileira).

Tabela 5. Ranking da produção científica das $\mathbf{5 0}$ primeiras instituições no mundo na área de Neurociências e Comportamento com artigos vinculados ao tema Educação - 1945 a 2014

\begin{tabular}{|c|c|c|}
\hline Rank. & IES & $\mathbf{N}^{\circ}$ de Documentos \\
\hline 1 & University of California System (EUA) & 798 \\
\hline 2 & Harvard University (EUA) & 420 \\
\hline 3 & Columbia University (EUA) & 395 \\
\hline 4 & University of London (Inglaterra) & 374 \\
\hline 5 & University of Michigan System (EUA) & 328 \\
\hline 6 & $\begin{array}{l}\text { Pennsylvania Commonwealth System of Higher } \\
\text { Education PCSHE (EUA) }\end{array}$ & 302 \\
\hline 7 & University of Toronto (Canadá) & 292 \\
\hline 8 & Johns Hopkins University (EUA) & 290 \\
\hline 9 & University of California Los Angeles (EUA) & 267 \\
\hline 10 & $\begin{array}{l}\text { Institut National de la Sante et de la Recherche } \\
\text { Medicale INSERM (França) }\end{array}$ & 265 \\
\hline 11 & University of California San Diego (EUA) & 241 \\
\hline 12 & Florida State University System (EUA) & 232 \\
\hline 13 & Mayo Clinic (EUA) & 231 \\
\hline 14 & University of Washington (EUA) & 231 \\
\hline 15 & University of Pittsburgh (EUA) & 230 \\
\hline 16 & University of Washington Seattle (EUA) & 230 \\
\hline 17 & University of California San Francisco (EUA) & 209 \\
\hline 18 & Rush University (EUA) & 202 \\
\hline 19 & University of Pennsy/vania (EUA) & 195 \\
\hline
\end{tabular}


Haeffner e Guimarães / Produção científica indexada na base Web of Science na área de Neurociências e Comportamento relacionada com

\begin{tabular}{|c|c|c|}
\hline Rank. & IES & $\mathbf{N}^{\circ}$ de Documentos \\
\hline 20 & University College London (EUA) & 194 \\
\hline 21 & Karolinska Institutet (Suécia) & 193 \\
\hline 22 & National Institutes of Health NIH (EUA) & 190 \\
\hline 23 & Duke University (EUA) & 189 \\
\hline 24 & Seoul National University (Coreia do Sul) & 178 \\
\hline 25 & Kings College London (Inglaterra) & 166 \\
\hline 26 & Oregon University System (EUA) & 163 \\
\hline 27 & $\begin{array}{l}\text { Centre National de la Recherche Scientifique CNRS } \\
\text { (França) }\end{array}$ & 161 \\
\hline 28 & Vu University Amsterdam (Holanda) & 148 \\
\hline 29 & US Department of Veteran Affairs (EUA) & 143 \\
\hline 30 & State University of New York Suny System (EUA) & 142 \\
\hline 31 & University of North Carolina (EUA) & 142 \\
\hline 32 & Boston University (EUA) & 141 \\
\hline 33 & Maastricht University (Holanda) & 140 \\
\hline 34 & University of Illinois Chicago (EUA) & 140 \\
\hline 35 & Mcgill University (Canadá) & 136 \\
\hline 36 & Baylor College of Medicine (EUA) & 135 \\
\hline 37 & Rutgers State University (EUA) & 135 \\
\hline 38 & University of British Columbia (EUA) & 135 \\
\hline 39 & New York University (EUA) & 128 \\
\hline 40 & Indiana University System (EUA) & 127 \\
\hline 41 & University of Helsinki (Finlândia) & 125 \\
\hline 42 & Yeshiva University (EUA) & 124 \\
\hline 43 & Stanford University (EUA) & 123 \\
\hline 44 & University of Southern California (EUA) & 123 \\
\hline 45 & Universidade de São Paulo (Brasil) & 122 \\
\hline 46 & University of Montreal (Canadá) & 121 \\
\hline 47 & University of Wisconsin System (EUA) & 121 \\
\hline 48 & University of lowa (EUA) & 119 \\
\hline 49 & University of Kansas (EUA) & 119 \\
\hline \multirow[t]{2}{*}{50} & Northwestern University (EUA) & 118 \\
\hline & Dados da Tabela & 10.173 \\
\hline
\end{tabular}

Fonte: Web of Science, Thomson Reuters. Acesso em: 10 maio 2015. 
Haeffner e Guimarães / Produção científica indexada na base Web of Science na área de Neurociências e Comportamento relacionada com o tema Educação

Analisando o ranking das instituições brasileiras (Tabela 6), os primeiros lugares estão localizados no estado de São Paulo, com a USP (122 documentos) em primeiro lugar e a Unifesp em segundo (63 documentos). Em seguida, Minas Gerais, com a UFMG (31 documentos), e Rio de Janeiro, com a UFRJ (29 documentos). Juntas, as 31 instituições nacionais na área de Neurociências e Educação (também majoritariamente universidades) produziram 482 artigos, representando $78,6 \%$ da produção brasileira (613) no período.

Tabela 6. Ranking da produção científica das primeiras instituições brasileiras na área de Neurociências e Educação: 1990-2014

\begin{tabular}{|c|c|c|}
\hline Rank. & Instituição & $\begin{array}{l}\text { Número de } \\
\text { Documentos }\end{array}$ \\
\hline 1 & Universidade de São Paulo (USP) & 122 \\
\hline 2 & Universidade Federal de São Paulo (Unifesp) & 63 \\
\hline 3 & Universidade Federal de Minas Gerais (UFMG) & 31 \\
\hline 4 & Universidade Federal do Rio de Janeiro (UFRJ) & 29 \\
\hline 5 & Universidade Estadual de Campinas (Unicamp) & 28 \\
\hline 6 & Universidade Federal do Rio Grande do Sul (UFRGS) & 28 \\
\hline 7 & $\begin{array}{l}\text { Pontifícia Universidade Católica do Rio Grande do Sul } \\
\text { (PUCRS) }\end{array}$ & 17 \\
\hline 8 & Rede Sarah de Hospitais de Reabilitação & 13 \\
\hline 9 & Universidade Federal de Santa Catarina (UFSC) & 11 \\
\hline 10 & Universidade Federal Fluminense (UFF) & 11 \\
\hline 11 & Universidade de Brasília (UnB) & 9 \\
\hline 12 & $\begin{array}{l}\text { Universidade Estadual Paulista Júlio de Mesquita } \\
\text { Filho (Unesp) }\end{array}$ & 9 \\
\hline 13 & Universidade Federal do Paraná (UFPR) & 9 \\
\hline 14 & Universidade Federal do Rio Grande do Norte (UFRN) & 9 \\
\hline 15 & $\begin{array}{l}\text { Assistência à Saúde de Pacientes com Epilepsia } \\
\text { (Aspe) }\end{array}$ & 7 \\
\hline 16 & Fundação Oswaldo Cruz (Fiocruz) & 7 \\
\hline 17 & Universidade do Estado do Rio de Janeiro (Uerj) & 7 \\
\hline 18 & Universidade Federal de Pernambuco (UFPE) & 7 \\
\hline 19 & $\begin{array}{l}\text { Universidade Federal do Estado do Rio de Janeiro } \\
\text { (UniRio) }\end{array}$ & 7 \\
\hline 20 & Hospital Israelita Albert Einstein & 6 \\
\hline
\end{tabular}


Haeffner e Guimarães / Produção científica indexada na base Web of Science na área de Neurociências e Comportamento relacionada com

\begin{tabular}{|c|l|c|}
\hline Rank. & \multicolumn{1}{|c|}{ Instituição } & $\begin{array}{c}\text { Número de } \\
\text { Documentos }\end{array}$ \\
\hline 21 & $\begin{array}{l}\text { Pontifícia Universidade Católica de Campinas (PUC- } \\
\text { Campinas) }\end{array}$ & 6 \\
\hline 22 & Universidade de Caxias do Sul (UCS) & 5 \\
\hline 23 & Faculdade de Medicina de Catanduva & 5 \\
\hline 24 & $\begin{array}{l}\text { Pontifícia Universidade Católica do Rio de Janeiro } \\
\text { (PUC-Rio) }\end{array}$ & 5 \\
\hline 25 & $\begin{array}{l}\text { Universidade Federal de Ciências da Saúde de Porto } \\
\text { Alegre (UFCSPA) }\end{array}$ & 5 \\
\hline 26 & Universidade Federal de São Carlos (UFSCar) & 4 \\
\hline 27 & Hospital Santa Marcelina & 4 \\
\hline 28 & Universidade Estadual de Londrina (UEL) & 4 \\
\hline 29 & Universidade Federal da Bahia (Ufba) & $\mathbf{4 8 2}$ \\
\hline 30 & Universidade Federal do ABC (UFABC) & $\mathbf{1 3 1}$ \\
\hline 31 & Universidade Federal do Ceará (UFC) & Total da Tabela \\
\hline & Outras Instituições & \\
\hline
\end{tabular}

Fonte: Web of Science, Thomson Reuters. Acesso em: 10 maio 2015.

Os dados da Tabela 6 apontam para um elevado índice de dupla contagem (85\%) dos artigos brasileiros nessa área. Essa proporção é 4,7 vezes mais elevada do que o índice mundial (18,5\%, Tabela 1), indicando a existência de forte cooperação entre os grupos de pesquisa das instituições do país. Essa percepção se torna ainda mais pertinente quando se observa o fato de ser reduzido o número dos grupos de pesquisa atuantes nesta área no Brasil, registrados no Diretório dos Grupos de Pesquisa do CNPq.

\section{Grupos de pesquisa}

Um total de 27 instituições acadêmicas contabiliza, ao todo, 35 grupos de pesquisa da área registrados no Diretório dos Grupos de Pesquisa do CNPq (Tabela 7). Ocupam as primeiras posições a Unifesp e a UFPB (com três grupos cada), seguidas de três instituições gaúchas, a PUCRS, a UFRGS e o IFSul, e uma paulista, a UFABC, todas com dois grupos cada. 
Haeffner e Guimarães / Produção científica indexada na base Web of Science na área de Neurociências e Comportamento relacionada com o tema Educação

Tabela 7. Número de grupos de pesquisa em Neurociências e Educação registrados no CNPq, por instituição

\begin{tabular}{|c|c|c|}
\hline Rank. & Instituição & Total \\
\hline 1 & Universidade Federal da Paraíba (UFPB) & 3 \\
\hline 2 & Universidade Federal de São Paulo (Unifesp) & 3 \\
\hline 3 & Instituto Federal Sul-Rio-Grandense (IFSul) & 2 \\
\hline 4 & Pontifícia Universidade Católica do Rio Grande do Sul (PUCRS) & 2 \\
\hline 5 & Universidade Federal do ABC (UFABC) & 2 \\
\hline 6 & Universidade Federal do Rio Grande do Sul (UFRGS) & 2 \\
\hline 7 & Centro Universitário FIEO & 1 \\
\hline 8 & $\begin{array}{l}\text { Faculdade de Ciências Médicas da Santa Casa de São Paulo } \\
\text { (FCMSCSP) }\end{array}$ & 1 \\
\hline 9 & Instituto Federal do Ceará (IFCE) & 1 \\
\hline 10 & Pontifícia Universidade Católica de Minas Gerais (PUC Minas) & 1 \\
\hline 11 & Universidade de São Paulo (USP) & 1 \\
\hline 12 & Universidade do Estado da Bahia (Uneb) & 1 \\
\hline 13 & Universidade do Estado de Minas Gerais (UEMG) & 1 \\
\hline 14 & Universidade do Estado do Amazonas (UEA) & 1 \\
\hline 15 & Universidade Estadual de Campinas (Unicamp) & 1 \\
\hline 16 & Universidade Estadual de Feira de Santana (UEFS) & 1 \\
\hline 17 & Universidade Estadual do Piauí (UFPI) & 1 \\
\hline 18 & Universidade Federal de Alagoas (Ufal) & 1 \\
\hline 19 & Universidade Federal de Juiz de Fora (UFJF) & 1 \\
\hline 20 & Universidade Federal de Minas Gerais (UFMG) & 1 \\
\hline 21 & Universidade Federal de Santa Catarina (UFSC) & 1 \\
\hline 22 & Universidade Federal do Maranhão (UFMA) & 1 \\
\hline 23 & Universidade Federal do Rio de Janeiro (UFRJ) & 1 \\
\hline 24 & Fundação Universidade do Rio Grande (Furg) & 1 \\
\hline 25 & Universidade Federal Fluminense (UFF) & 1 \\
\hline 26 & Universidade Presbiteriana Mackenzie (UPM) & 1 \\
\hline 27 & Universidade Tiradentes (UNIT) & 1 \\
\hline Total geral & & 35 \\
\hline
\end{tabular}

Fonte: CNPq. Diretório dos Grupos de Pesquisa no Brasil. Disponivel em: <http://dgp.cnpq.br/dgp/faces/consulta/consulta_ parametrizada.jsf>. Acesso em: 25 jun. 2015.

A Tabela 8 mostra que um total de 197 pesquisadores atuantes nessa área de pesquisa estão distribuídos em diversas grandes áreas 
do conhecimento, especialmente nas Ciências Humanas, na Saúde e nas Ciências Biológicas, sendo predominante o pessoal com titulação no doutorado (cerca de $50 \%$ ) e no mestrado $(23 \%$ ) e com treinamento pós-doutoral (23\%).

Tabela 8. Número de pesquisadores em grupos de pesquisa em Neurociências e Educação por grande área e formação acadêmica

\begin{tabular}{|c|l|c|c|c|c|c|c|}
\hline Nr. & $\begin{array}{c}\text { Formação } \\
\text { acadêmica }\end{array}$ & $\begin{array}{c}\text { Ciências } \\
\text { Biológicas }\end{array}$ & $\begin{array}{c}\text { Ciências } \\
\text { da Saúde }\end{array}$ & $\begin{array}{c}\text { Ciências } \\
\text { Exatas e } \\
\text { da Terra }\end{array}$ & $\begin{array}{c}\text { Ciências } \\
\text { Humanas }\end{array}$ & $\begin{array}{c}\text { Linguística, } \\
\text { Letras e Artes }\end{array}$ & $\begin{array}{c}\text { Total } \\
\text { geral }\end{array}$ \\
\hline 1 & Doutorado & 17 & 21 & 10 & 47 & 3 & 98 \\
\hline 2 & Pós-doutorado & 13 & 10 & 12 & 10 & 1 & 46 \\
\hline 3 & Mestrado & 6 & 9 & 2 & 27 & 1 & 45 \\
\hline 4 & Especialização & - & - & - & 4 & - & 4 \\
\hline 5 & Graduação & - & - & - & 2 & - & 2 \\
\hline 6 & $\begin{array}{l}\text { Mestrado } \\
\text { profissional }\end{array}$ & - & 1 & - & 1 & - & 2 \\
\hline Total & & $\mathbf{3 6}$ & $\mathbf{4 1}$ & $\mathbf{2 4}$ & $\mathbf{9 1}$ & $\mathbf{5}$ & $\mathbf{1 9 7}$ \\
\hline
\end{tabular}

Fonte: CNPq. Diretório dos Grupos de Pesquisa no Brasil. Disponível em: <http://dgp.cnpq.br/dgp/faces/consulta/consulta_ parametrizada.jsf>. Acesso em: 25 jun. 2015.

Os grupos de pesquisa da área incorporam também um grande contingente de estudantes (Tabela 9), um total de 315, sendo: ensino médio (35\%), graduação (24\%), especialização (14\%) e mestrado (13\%). Esse contingente encontra-se concentrado em maior proporção na grande área de Ciências Humanas, à qual a área de Educação é vinculada, indicando que esta é a área que mais se destaca quanto à existência de grupo de pesquisa que vincula Neurociências e Educação.

Tabela 9. Número de estudantes vinculados aos grupos de pesquisa em Neurociências e Educação por grande área e formação acadêmica

\begin{tabular}{|c|l|c|c|c|c|c|c|}
\hline Nr. & \multicolumn{1}{|c|}{$\begin{array}{c}\text { Formação } \\
\text { acadêmica }\end{array}$} & $\begin{array}{c}\text { Ciências } \\
\text { Biológicas }\end{array}$ & $\begin{array}{c}\text { Ciências } \\
\text { da Saúde }\end{array}$ & $\begin{array}{c}\text { Ciências } \\
\text { Exatas e } \\
\text { da Terra }\end{array}$ & $\begin{array}{c}\text { Ciências } \\
\text { Humanas }\end{array}$ & $\begin{array}{c}\text { Linguística, } \\
\text { Letras e Artes }\end{array}$ & $\begin{array}{c}\text { Total } \\
\text { geral }\end{array}$ \\
\hline 1 & $\begin{array}{l}\text { Ensino médio } \\
\left(2^{\circ} \text { grau }\right)\end{array}$ & 15 & 23 & 5 & 45 & 22 & 110 \\
\hline 2 & Graduação & 28 & 14 & 3 & 29 & 3 & 77 \\
\hline 3 & Especialização & 11 & 8 & - & 25 & - & 44 \\
\hline 4 & Mestrado & 13 & 14 & - & 15 & - & 42 \\
\hline 5 & Outros & 1 & 5 & - & 8 & 2 & 16 \\
\hline 6 & $\begin{array}{l}\text { Ensino } \\
\text { profissional de } \\
\text { nivel técnico }\end{array}$ & 2 & 1 & 1 & 2 & - & 6 \\
\hline
\end{tabular}


Haeffner e Guimarães / Produção científica indexada na base Web of Science na área de Neurociências e Comportamento relacionada com o tema Educação

\begin{tabular}{|c|c|c|c|c|c|c|c|}
\hline Nr. & $\begin{array}{l}\text { Formação } \\
\text { acadêmica }\end{array}$ & $\begin{array}{l}\text { Ciências } \\
\text { Biológicas }\end{array}$ & $\begin{array}{l}\text { Ciências } \\
\text { da Saúde }\end{array}$ & $\begin{array}{l}\text { Ciências } \\
\text { Exatas e } \\
\text { da Terra }\end{array}$ & $\begin{array}{l}\text { Ciências } \\
\text { Humanas }\end{array}$ & $\begin{array}{l}\text { Linguística, } \\
\text { Letras e Artes }\end{array}$ & $\begin{array}{l}\text { Total } \\
\text { geral }\end{array}$ \\
\hline 7 & $\begin{array}{l}\text { Extensão } \\
\text { universitária }\end{array}$ & 2 & 2 & - & 1 & - & 5 \\
\hline 8 & $\begin{array}{l}\text { Curso de curta } \\
\text { duração }\end{array}$ & 1 & 2 & - & 1 & - & 4 \\
\hline 9 & $\begin{array}{l}\text { Ensino } \\
\text { fundamental } \\
\left.\text { ( } 1^{\circ} \mathrm{grau}\right)\end{array}$ & - & - & - & 4 & - & 4 \\
\hline 10 & Doutorado & 1 & - & - & 2 & - & 3 \\
\hline 11 & Pós-doutorado & 2 & - & - & - & - & 2 \\
\hline 12 & $\begin{array}{l}\text { Residência } \\
\text { médica }\end{array}$ & - & 1 & - & - & - & 1 \\
\hline 13 & $\begin{array}{l}\text { Mestrado } \\
\text { profissional }\end{array}$ & - & - & - & 1 & - & 1 \\
\hline Total & & 76 & 70 & 9 & 133 & 27 & 315 \\
\hline
\end{tabular}

Fonte: CNPq. Diretório dos Grupos de Pesquisa no Brasil. Disponivel em: <http://dgp.cnpq.br/dgp/faces/consulta/consulta_ parametrizada.jsf>. Acesso em: 25 jun. 2015

Nesse sentido, tornou-se importante verificar como está no Brasil a pós-graduação nessa temática. Em pesquisa realizada nos sites da Capes, GeoCapes e Plataforma Sucupira, foram identificados 41 programas de pós-graduação em Neurociências (programas com o termo "neuro" no nome, na área de concentração e/ou na linha de pesquisa) em funcionamento, não foram encontradas informações sobre cursos de pós-graduação vinculando totalmente Neurociências e Educação. Foi, então, realizada uma busca pelo termo "educ*" nos sites dos programas que possuem o termo "neuro*" no nome do curso ou nas áreas de concentração, nas linhas de pesquisa e/ou ainda como área básica, e, novamente, nenhuma informação positiva foi recuperada. Uma nova busca foi feita, buscando verificar nos sites desses programas, e foram encontrados 16 programas que possuem na descrição (em seus respectivos sites) do programa, das disciplinas, das linhas ou projetos de pesquisa as palavras "aprendizado", "aprendizagem", "neuroplasticidade”, "neuroeducação" e "cognição", por estar subentendido que têm uma ligação com a educação, termo que não foi encontrado na pesquisa inicial.

Como indicado na Tabela 10, sete programas estão na região Sudeste (quatro em São Paulo, dois no Rio de Janeiro e um em Minas Gerais), cinco na região Sul (três no Rio Grande do Sul, um no Paraná e um em Santa Catarina); dois na região Norte (ambos no Pará) e dois 
no Nordeste (um no Rio Grande do Norte e outro na Paraíba); em relação à área de avaliação à qual estão vinculados, seis são da área de Ciências Biológicas II, três da Psicologia e Medicina II, dois da Medicina I e Interdisciplinar. Em sua grande maioria (14 no total), os programas possuem o nível de mestrado e de doutorado, somente dois possuem apenas o nível de mestrado. Merece destaque o fato de que quatro são programas de excelência: dois com nota 7 e dois com nota 6. Destaquese também que somente o programa Neurociência e Cognição da UFABC possuía palavra "neuroeducação" na sua descrição.

Conclui-se, portanto, que a formação de recursos humanos qualificados no nível da pós-graduação na temática NeurociênciaEducação está defasada em relação ao que ocorre na maioria das áreas da pós-graduação no Brasil.

Tabela 10. Programas em funcionamento em Neurociências que possuem na descrição (em seus respectivos sites) do programa, disciplinas, linhas ou projetos de pesquisa as palavras "aprendizado", "aprendizagem", "neuroplasticidade", "neuroeducação" e "cognição"

\begin{tabular}{|c|l|l|c|c|c|}
\hline Nr. & Sigla & \multicolumn{1}{|c|}{ Nome programa } & Área de avaliação & Nivel & Nota \\
\hline 1 & PUC/RS & Medicina e Ciências da Saúde & Medicina I & M/D & 7 \\
\hline 2 & Unifesp & Psicobiologia & Medicina II & M/D & 7 \\
\hline 3 & UFRGS & Ciências Biológicas (Bioquímica) & Ciências Biológicas II & M/D & 6 \\
\hline 4 & UFRJ & Ciências Morfológicas & Ciências Biológicas II & M/D & 6 \\
\hline 5 & UFMG & Neurociências & Interdisciplinar & M/D & 5 \\
\hline 6 & UFRGS & Ciências Biológicas (Neurociências) & Ciências Biológicas II & M/D & 5 \\
\hline 7 & Unicamp & Ciências Médicas & Medicina I & M/D & 5 \\
\hline 8 & UFABC & Neurociência e Cognição & Interdisciplinar & M/D & 4 \\
\hline 9 & UFF & Medicina (Neurologia) & Medicina II & M/D & 4 \\
\hline 10 & UFPA & Neurociências e Biologia Celular & Ciências Biológicas II & M/D & 4 \\
\hline 11 & UFPR & Saúde da Criança e do Adolescente & Medicina II & M/D & 4 \\
\hline 12 & UFRN & Neurociências & Ciências Biológicas II & M/D & 4 \\
\hline 13 & UFSC & Neurociências & Ciências Biológicas II & M/D & 4 \\
\hline 14 & USP & Neurociências e Comportamento & Psicologia & M/D & 4 \\
\hline 15 & UFPA & Neurociências e Comportamento & Psicologia & M & 3 \\
\hline 16 & UFPB/J.P. & Neurociência Cognitiva e Comportamento & Mia & 3 \\
\hline
\end{tabular}

Fonte: elaborada pelos autores com base nos sites GeoCapes e Plataforma Sucupira, da Capes. 
Haeffner e Guimarães / Produção científica indexada na base Web of Science na área de Neurociências e Comportamento relacionada com o tema Educação

\section{Considerações finais}

Os muitos estudos das Neurociências têm mostrado, cada vez mais, a importância dos componentes neurofisiológicos na formação dos processos cognitivos e socioemocionais das crianças, com reflexos na etapa inicial da educação infantil e posterior desdobramento no desempenho dos indivíduos na vida adulta. Assim, apesar de constituir área de estudos relativamente recente, a conexão NeurociênciasEducação tem mostrado assumir crescente relevância no cenário educacional em todo o mundo. Com efeito, tais estudos apontam para uma conexão promissora, englobando estudos sistemáticos que adotam metodologia não invasiva para obter informações e gerar conhecimentos sobre aspectos cognitivos do processo educacional (DE PONTES VIEIRA, 2013), vale dizer, de aprendizagem e memorização ainda na fase infantil. Na verdade, é na infância e mesmo na fase embrionária da vida humana que se formam muitos dos circuitos neuronais que vão constituir o cérebro racional do futuro cidadão na fase adulta. Saber como os impulsos externos dão constituição e são iniciadores do processo de aprendizagem das crianças é um campo de pesquisa promissor para a formatação de modelos educacionais baseados em dados científicos seguros capazes de orientar a relação ensino-aprendizagem.

A conexão mais importante aqui é a que extrai da pesquisa científica, a partir de experimentos com metodologia apropriada das Neurociências, os resultados capazes de levar à prática do processo educacional mais apropriado e eficiente (GOSWAMI, 2006), constituindo a ponte necessária capaz de gerar significativos avanços na área educacional (SIGMAN et al., 2014). Outros autores sugerem que os resultados dos estudos oriundos da Neurociência quando aplicados à educação podem constituir a "parceria ideal para produzir soluções baseadas em evidência capazes de constituir um guia para o ensino no século 21" (CAREW; MAGSAMEN, 2010). A apropriação dos conhecimentos gerados na parceria Neurociências-Educação, por seus componentes tecnológico e econômico, vinculando ao desenvolvimento da capacitação humana (HECKMAN, 2007; HECKMAN, 2011), levou à concessão do Prêmio Nobel ao economista James J. Heckman, no ano 2000. 
Haeffner e Guimarães / Produção científica indexada na base Web of Science na área de Neurociências e Comportamento relacionada com

Neste trabalho buscou-se mostrar as principais características da produção científica em Neurociências e Comportamento vinculada ao tema Educação. Foi possivel mapear os países e as instituições onde esta pesquisa é feita, em quais periódicos ela é publicada e onde estão os grupos de pesquisa brasileiros que a desenvolvem no país.

No Brasil, estudos sobre a conexão Neurociências-Educação têm também despertado interesse e motivado debates, seminários e eventos, visando discutir e analisar a influência de tais experiências na formação e no trabalho docente (AMARAL; JANDREY, 2014, 2015). A confirmação do crescente interesse na temática de pesquisa Neurociências-Educação é mostrada pelo crescimento do número de publicações sobre o tema em todo o mundo e também no Brasil.

Como mostrado neste trabalho, os dados brasileiros são ainda pouco expressivos do ponto de vista quantitativo, tanto no número de publicações como no de grupos de pesquisa e, consequentemente, no sistema de formação e de capacitação de recursos humanos, sobretudo na pós-graduação. Também no que concerne ao diálogo entre neurocientistas e professores da educação básica, não há indícios da existência da colaboração mútua na pesquisa que precisaria existir em grau de participação efetivamente mais elevado. Por um lado, a interação entre os grupos das instituições brasileiras na pesquisa sobre as relações Neurociências e Educação dá forte indício da percepção da importância do tema no Brasil, mas, por outro, o baixo índice de colaboração internacional constitui deficiência a ser corrigida.

Recebido em 20/11/2015

Aprovado em 07/04/2016 


\section{Referências}

AMARAL, J. H.; JANDREY, C. M. A Educação no "século do cérebro": estudo sobre a aproximação entre Neurociências e Educação no Brasil. In: SEMINÁRIO DE PESQUISA EM EDUCAÇÃO DA REGIÃO SUL - ANPED-SUL, 10., 2014, Florianópolis. Anais... Florianópolis, Brasil: ANPED-SUL, 2014.

Neuroeducação e as reivindicações de cientificidade para a pesquisa educacional e o trabalho docente. In: SEMINÁRIO BRASILEIRO SEMINÁRIO INTERNACIONAL DE ESTUDOS CULTURAIS E EDUCAÇÃO, 3., 2015, Canoas. Anais... Canoas: Universidade Luterana do Brasil, 2015.

BARTOSZECK, A. B. Neurociência na educação. Revista Eletrônica Faculdades Integradas Espírita, v. 1, p. 1-6, 2006.

CAREW, T. J.; MAGSAMEN, S. H. Neuroscience and education: An ideal partnership for producing evidence-based solutions to guide 21st century learning. Neuron, San Diego, v. 67, n. 5, p. 685-688, 2010.

DE PONTES VIEIRA, E. P. Neurociências, Cognição e Educação: Limites e Possibilidades na Formação de Professores. Revista Práxis, Volta Redonda, v. 4, n. 8, 2013.

GOSWAMI, U. Neuroscience and education: from research to practice? Nature Reviews Neuroscience, Londres, v. 7, n. 5, p. 406-413, 2006.

HAEFFNER, C. Condições sócio-econômicas das nações refletidas na produção científica: um estudo exploratório entre Brasil e Coréia do Sul. 2006. Trabalho de conclusão (graduação em Biblioteconomia)-Faculdade de Biblioteconomia e Comunicação, Universidade Federal do Rio Grande do Sul, Porto Alegre, RS, 2006.

HECKMAN, J. J. The economics, technology, and neuroscience of human capability formation. Proceedings of the national Academy of Sciences, Washington, v. 104, n. 33, p. 13.250-13.255, 2007.

The Economics of Inequality: The Value of Early Childhood

Education. American Educator. v. 35, n. 1, p. 31-47, 2011. 
Haeffner e Guimarães / Produção científica indexada na base Web of Science na área de Neurociências e Comportamento relacionada com HOWARD-JONES, P. A. Neuroscience and education: myths and messages. Nature Reviews Neuroscience, Londres, v. 15, p. 817-824, 2014.

MEIS, L.; LETA, J. O Perfil da ciência brasileira. Rio de Janeiro: Editora UFRJ, 1996.

SIGMAN, M. et al. Neuroscience and education: prime time to build the bridge. Nature Neuroscience, Nova lorque, v. 17, n. 4, p. 497-502, 2014.

SILVA, J. A.; BIANCHI, M. L. P. Cientometria: a métrica da ciência. Paidéia, Ribeirão Preto, v. 11, n. 21, p. 5-10, 2001.

THOMSON REUTERS. Indicators Handbook. Philadelphia: Thomson Reuters, 2015. 